\title{
Einwanderungspolitik und öffentliche Meinung in Argentinien 1933-1945
}

\author{
Carlota Jackisch
}

Der Aufstieg des Nationalsozialismus in Deutschland brachte die Errichtung eines totalitären Regimes und damit den Ausschließlichkeitsanspruch einer Partei auf die Führung mit sich. Sobald das Einparteiensystem eingeführt und eine Politik der Rassentrennung entwickelt worden waren, gab es zwei Gruppen, die unbarmherzig verfolgt wurden: politisch Andersdenkende und Juden - aber auch Mitglieder verschiedener religiöser Institutionen, ethnische Gruppen, wie z. B. Zigeuner, und Geisteskranke wurden diskriminiert und verfolgt. Für sie war kein Platz mehr in Deutschland. Wer konnte, floh ins Exil, einige unter ihnen nach Argentinien.

Schon bevor über Hunderttausende von Menschen diese Tragödie hereinbrach, kam es in Argentinien zu einem Wandel der politischen Landschaft, der sich inhaltlich negativ für jene auswirken sollte, die auf der Flucht aus Deutschland nach Argentinien einwandern wollten. So beendete der Staatsstreich, mit dem im September 1930 Präsident Hipólito Yrigoyen gestürzt wurde, eine ununterbrochene, fast 70jährige Abfolge von konstitutionellen Präsidenten und praktisch zwei Jahrzehnte echter Demokratie. In den 30er Jahren gab Argentinien somit den politischen Kurs auf, der die Entwicklung des Landes seit dem Ende des 19. Jahrhunderts bestimmt hatte, ohne jedoch die Konsolidierung eines rechtmäßigen politischen Systems und ebensowenig eine gewisse Kohärenz in den Regierungsentscheidungen zu erreichen. Dieser Mangel an politischer Konsistenz wurde u. a. deutlich anhand der argentinischen Innenpolitik jener Zeit, deren genaue Analyse hier zu weit führen würde, sowie anhand der Reaktion der Regierung auf die Haltung der argentinischen Öffentlichkeit gegenüber dem Nationalsozialismus und dem Schicksal der Flüchtlinge aus dem Dritten Reich.

Die zunehmend restriktivere Einwanderungspolitik einerseits und ihre tatsächlichen Auswirkungen andererseits stellen in dieser Hinsicht ein klares Beispiel für jene inkonsequente Politik dar.

\section{Einwanderungspolitik 1933-1945}

Bereits vor 1933 zeichnete sich in der Einwanderungspolitik eine Tendenz ab, die sich in der Einführung von Einwanderungsbeschränkungen äußerte und folglich zeitlich nichts mit Rassenvorurteilen oder politischen Befürchtungen zu tun haben konnte, welche aus den politischen Vorkommnissen in Europa im hier betrachteten Zeitraum 1933-1945 resultierten. So beschlossen die De-factoMachthaber schon 1930, nach dem Sturz der verfassungsmäßigen Regierung unter Präsident Hipólito Yrigoyen, eine Erhöhung der Stempelgebühren für die 
Ausstellung der für die Einreise benötigten Visa und Unterlagen. Daneben wurden die argentinischen Konsulate angewiesen, jede Art der Förderung der Immigration zu vermeiden und jene, die trotz dieser Hindernisse einwandern wollten, vor den Schwierigkeiten auf dem argentinischen Arbeitsmarkt zu warnen. Es verwundert nicht, daß die autoritären Elemente, die den Boden für einen Militärputsch bereitet hatten, welcher der relativ stabilen demokratischen Tradition Argentiniens jener Jahre ein Ende machte, auch mit der Politik der offenen Grenzen brechen wollten, die das Land bis dahin vertreten hatte und die auf den liberalen Grundsätzen der argentinischen Verfassung basierte. Die zunehmend restriktiveren Einwanderungsgesetze Argentiniens, die die Politik der 30er Jahre prägten, sind als Teil einer weitreichenden Aufgabe von Werten zu sehen, die in großem Maße das argentinische Leben bis dahin bestimmt hatten.

Seitdem der Staat ab 1876 mit beträchtlicher Unterstützung der Privatwirtschaft eine klare Pro-Immigrationspolitik betrieb, verwandelte der konstante Zustrom von Immigranten verschiedener Nationalitäten Argentinien in ein Land, in dem in einigen Regionen - betrachtet man bestimmte Altersgruppen - die Mehrheit der Bevölkerung aus Ausländern bestand. In den 30er Jahren aber entschied man sich für eine politische Kursänderung: Man begann, die Grenzen des Landes zu schließen, die demokratische Legitimität wurde verleugnet, Wahlfälschungen und das Eingreifen der Armee in das politische Geschehen sowie das Erstarken nationalistischer Gruppierungen wurden toleriert.

$\mathrm{Da}$ diese Tendenzen zeitlich vor jenen Ereignissen anzusiedeln sind, die die zwangsweise Einwanderung von Juden und Gegnern des Nationalsozialismus aus Deutschland verursachen sollten - wobei sie aber im Kontext eines zu autoritären Regierungen neigenden weltpolitischen Klimas zu sehen sind -, zeigt die Tatsache, daß im nationalen argentinischen Abgeordnetenhaus schon 1933 ein Gesetzentwurf vorgelegt wurde, der für fünf Jahre ein absolutes Einreiseverbot für Ausländer, die arbeitssuchend ins Land kamen, vorsah. Wenn auch dieses Gesetz nicht verabschiedet wurde, so hatte man zu seiner Rechtfertigung doch die Existenz widriger wirtschaftlicher Umstände angeführt. Dieses Argument sollte von da ab als Vorwand für die Erlassung von Einwanderungsbeschränkungen dienen. Selbst als man schon Rassenvorurteile gegen diese spezielle Immigration hegte, wie vertrauliche Unterlagen des Außenministeriums belegen, schob man weiterhin wirtschaftliche Probleme als Grund vor.Wie schon in einer anderen Studie (Jackisch 1989) gezeigt wurde, halten die Befürchtungen hinsichtlich der Arbeitslosigkeit einer Prüfung jedoch nicht stand. Verglichen mit den Durchschnittszahlen der vorangegangenen Jahre nahm ab 1933 die Wirtschaftstätigkeit in Argentinien zu, und die vorhandene Arbeitslosigkeit war vergleichsweise gering.

1933 trat ein Dekret in Kraft, das laut seiner Begründung "zur Verteidigung der in Argentinien ansässigen Arbeiter" erlassen wurde, und das die argentinischen Konsuln im Ausland anwies, bis auf weitere Anordnungen keine Visa oder Einreisegenehmigungen für Immigranten auszustellen - gleich welcher 
Nationalität und aus welchem unmittelbaren Herkunftsland - , wenn diese nicht nachweisen konnten, daß sie einen Posten, eine Anstellung oder Beschäftigung, die ihnen den Lebensunterhalt garantieren könnten (Art. 1) in Aussicht hatten. Demgemäß mußte, wer als Einwanderer ins Land kommen wollte, einen Arbeitsvertrag oder eine Arbeitsvereinbarung vorweisen (Art. 2). Daneben wurde aber die Zentrale Einwanderungsbehörde ermächtigt, die Einreise von Großeltern, Eltern, Ehepartnern, Kindern, Geschwistern, Enkelkindern und blutsverwandten Neffen und Nichten von in Argentinien ansässigen Ausländern zu gestatten, vorausgesetzt, jene bürgten für deren Solvenz und einwandfreies Verhalten und verpflichteten sich, nötigenfalls den Unterhalt für den Neuankömmling zu bestreiten.

Im darauffolgenden Jahr schuf ein ergänzendes Dekret die Möglichkeit, u. a. auf Bitten von in Argentinien lebenden Angehörigen, eine Einreiseerlaubnis zu erhalten. Für die vorliegende Arbeit ist hierbei von besonderem Interesse, $\mathrm{da}$ der Verwandtschaftsgrad der Angehörigen, die ihre Verwandten "zu sich rufen" konnten, sehr verschieden war. So akzeptierte man Blutsverwandtschaften bis zum zweiten Grad in aufsteigender und absteigender Linie (Kinder und Enkelkinder/Eltern und Großeltern) und Verschwägerung ersten Grades (Schwiegersohn oder -tochter/Schwiegervater oder -mutter). Es bestand auch die Möglichkeit, eine "Erlaubnis zur freien Einreise" für einen Ehepartner auf Betreiben des anderen, bereits im Land lebenden Ehegatten zu erhalten. In gleicher Weise konnten von einem Onkel "gerufene" Neffen oder Nichten ins Land gelangen, und umgekehrt.

Die Aufzählung zeigt, daß diese Bestimmung für die Flüchtlinge des Dritten Reiches eine echte Einreisemöglichkeit nach Argentinien bedeutete, einerseits wegen der breiten Skala von Verwandten, die einen anderen Angehörigen, der Deutschland verlassen wollte, zu sich holen konnten, und andererseits weil dieser Weg durch das sehr restriktive Dekret aus dem Jahr 1938 nicht völlig verstellt wurde, wenngleich sein Umfang verglichen mit der Anfangszeit eingeschränkt wurde.

Als immer mehr Einwanderungsbeschränkungen erlassen wurden, d. h. immer mehr Unterlagen von jenen vorgelegt werden mußten, die ins Land einreisen wollten (von ausländischer Justizbehörde oder Polizei ausgestelltes Führungszeugnis, Nachweis der Nichtarmut, Gesundheitszeugnis), tat sich dennoch eine neue Lücke in den Einreisebestimmungen auf. 1934 befreite ein neues Dekret ("Regelung für reisende Touristen") jene, die das Land bereisen wollten, von der Vorlage einiger der genannten Dokumente, wodurch nur noch der Reisepaß und eine von einer argentinischen Konsularbehörde am Einschiffungsort ausgestellte Touristenbescheinigung vorgelegt werden mußten. Bereits während der ersten Jahre ermöglichte diese Verfügung die Einreise von zahlreichen deutschen Immigranten, die, einmal im Land, nun Verwandte zu sich holen konnten, wie oben erwähnt wurde.

Daß die Möglichkeit, dank einer Erste-Klasse-Passage ins Land zu kommen, von denen, die Deutschland verlassen mußten, sehr oft in Anspruch genommen 
wurde, beweist der extreme Anstieg der Zahl Deutscher, die mit einer Passage solcher Art einreisten. Das Besondere an dieser Situation wird umso deutlicher, setzt man die Zahl der Erste-Klasse-Reisenden mit der der übrigen Klassen in Relation. Betrachtet man die dem Jahr 1933 vorangegangenen zehn Jahre, so liegt der Anteil der deutschen Erste-Klasse-Reisenden verglichen mit der Gesamtzahl Reisender aus Deutschland zwischen 12 und $13 \%$. In den Jahren 1933 bis 1941 schwankte dagegen dieser Anteil zwischen 24 und $46 \%$.

1938 hatte das Problem der Verfolgten des in Deutschland herrschenden Naziregimes bereits internationale Dimensionen erreicht, so daß die verschiedenen Länder, in die die Flüchtlinge aus Deutschland geflohen waren oder noch fliehen wollten, zu Entscheidungen gezwungen waren.

Im selben Jahr wurde die Konferenz von Evian mit dem Ziel einberufen, das Flüchtlingsproblem zu lösen und durch die Darlegung der Situation, unter der Juden und Gegner des Nationalsozialismus litten, das Gewissen der Teilnehmerstaaten wachzurufen. Doch erreichte man genau den entgegengesetzten Effekt (Avni 1983). Die Schilderung der großen Flüchtlingsströme auf der Suche nach irgendeinem Zufluchtsort rief keineswegs die gewünschte Reaktion hervor, im Gegenteil. Praktisch alle an der Konferenz teilnehmenden Länder schlossen von da an ihre Grenzen für Emigranten aus Deutschland. Auch Argentinien senkte 1938 die Schlagbäume, oder versuchte es zumindest, denn unter der oben erwähnten Kategorie "Tourist" reisten statistisch gesehen noch Personen ein, als der Krieg bereits eine Intensität erreicht hatte, die es aufgrund all der Gefahren einer solchen Überfahrt schwierig machte, eine normale Reise anzutreten.

Die gesetzliche Grundlage, die zur drastischen Verringerung der Zahl der nach Argentinien einreisenden Deutschen führte, bildete das Dekret 8972 vom 28. Juli 1938. In seiner Begründung wurde auf statistische Angaben Bezug genommen, die zeigen sollten, daß aufgrund von Trockenheit und anderen widrigen Naturereignissen die Agrarproduktion deutlich zurückgegangen war. Dies, so die Verfasser des Dekrets, führte in Folge zu einem Beschäftigungsrückgang bei Arbeitern und Tagelöhnern. Unter diesen Umständen könne sich der Zustrom von Immigranten für die in Argentinien lebenden Arbeiter enorm negativ auswirken. Des weiteren wurde erklärt, daß diese Maßnahme ergriffen werden müsse, "da die momentane internationale Lage einen direkten Anstieg von Immigranten vorausahnen läßt, die aus unerheblichen Motiven heraus auswandern wollen, was mit den Erfordernissen einer gesunden Einwanderungspolitik nicht zu vereinbaren ist".

Nun ist es lächerlich, in einem Land, in dem sich die Situation binnen wenigen Monaten ändern kann, zur Erklärung der Arbeitslosigkeit eine Dürreperiode als Argument anzuführen. Tatsächlich erbrachten Landwirtschaft und Viehzucht schon 1939 wieder ähnliche Erträge wie in den Jahren vor 1938. So läßt alles vermuten, daß die Absicht des Dekrets in Wirklichkeit darin lag, die Einreise von Juden nach Argentinien zu verhindern (Jackisch 1987), was später durch die Weisung an die argentinischen Diplomaten bestätigt wurde, die das 
Problem der Flüchtlinge des nationalsozialistischen Regimes vor internationalen Gremien vertreten sollten.

Ihren Höhepunkt erreichten die restriktiven Maßnahmen mit dem Dekret vom 17. September 1941, das einen aus drei Mitgliedern bestehenden Immigrationsrat einsetzte, der über die Einreise oder Ablehnung einer jeden Person Immigrant oder nicht - entscheiden konnte. In der Begründung des Dekrets war eindeutig bestimmt, daß es darum ging, die Einwanderung von Flüchtlingen und den Transit von Emigranten zu verhindern.

Im Gegensatz zur Härte der geschriebenen Normen bei jeglichen Einreisegenehmigungsverfahren für Immigranten fällt die Toleranz auf, die die Behörden an den Tag legten, wenn es um Einwanderer ging, die illegal über die Grenze gekommen oder im Land geblieben waren, nachdem sie als Touristen oder Transitreisende eingereist waren. Dies bedeutet, daß Argentinien es vorzog, einen "Infiltrationsprozeß" zu tolerieren, statt offen eine Zahl von Flüchtlingen des Dritten Reiches aufzunehmen, was einmal mehr die Inkohärenz beweist, die zu Anfang erwähnt wurde. Eine Fortsetzung der traditionellen Politik der offenen Grenzen für Immigranten hätte bedeutet, angesichts der Verfolgung, die im Hitler-Deutschland stattfand, im Einklang mit den konstitutionellen Grundsätzen des Landes Stellung zu beziehen. Argentinien zog es vor, diese Prinzipien außer acht zu lassen, trotz der vielen Stimmen, die in der argentinischen Öffentlichkeit laut wurden und den Nationalsozialismus verurteilten.

\section{Die öffentliche Meinung in Argentinien gegenüber Nationalsozialismus und Flüchtlingsproblem}

Die Aktivitäten nationalsozialistischer Gruppen in Argentinien setzten vor der Machtergreifung Hitlers ein. Ab 1933 erhielten diese noch in der Anfangsphase stehenden Organisationen Direktiven aus Deutschland hinsichtlich der Notwendigkeit, die Deutschen, welche außerhalb der Grenzen des Dritten Reiches lebten, in die nationalsozialistische Bewegung einzugliedern (Jacobsen 1968). Während der ersten Jahre hinderten die argentinischen Behörden die Nazigruppen in Argentinien nicht an ihrem Tun. Der argentinische Botschafter in Deutschland Labougle sagte Jahre später im Gespräch mit dem deutschen Staatssekretär im Auswärtigen Amt v. Weizsäcker, daß sich die nationalsozialistischen Aktivitäten von Beginn an ohne Probleme entwickeln konnten, "weil Argentinien ein Land ist, wo im allgemeinen ein jeder tut, was er will". ${ }^{1}$ Nach fünf Jahren ohne strafrechtliche Verfolgung begann das Politspektrum Argentiniens auf die nationalsozialistische Propaganda zu reagieren. Die im Abgeordnetenhaus repräsentierten politischen Kräfte vertraten gegenüber den nazistischen Machenschaften im Land - und sogar gegenüber der Verbreitung dieser ideologischen Strömung - verschiedene Ansichten. Deren feine Unterschiede

${ }^{1}$ E. Frhr. v. Weizsäcker 18.5.1938, A. A., Pol. IX, 759, Akten zur Deutschen Auswärtigen Politik, 1918-1945, 712. 
wurden in den Debatten deutlich, welche sich mit einigen Unterbrechungen über vier Jahre hinzogen.

Die Unión Cívica Radical (Radikale Bürgerunion) und die Sozialisten prangerten die nationalsozialistischen Aktivitäten im Land an, während sie gleichzeitig auch das in Deutschland herrschende Regime als Feind der Vernunft und des Rechts angriffen. Eine ähnliche Position vertraten die wichtigsten Zeitungen des Landes, so daß Botschafter v. Thermann seinem Ministerium in Deutschland mitteilte: "Die wirklich einflußreichen Zeitungen wie La Prensa, La Nación oder El Mundo sind nicht käuflich, nicht einmal für Millionenbeträ-

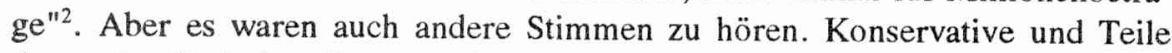
des nationalistischen Lagers neigten dazu, das Problem der nationalsozialistischen Einflußnahme zu bagatellisieren. Sie argumentierten, eine Untersuchung der Aktivitäten dieser Gruppen, wie sie Radikale und Sozialisten vorschlugen, könne die Beziehungen des Landes zu Deutschland beeinträchtigen.

Als Reaktion auf die kategorische Ablehnung der nationalsozialistischen Umtriebe im Land durch die öffentliche Meinung wurden drei Dekrete unterzeichnet, die diese Aktivitäten in gewisser Hinsicht einschränkten, zumindest was die nach außen wirkenden und für die argentinische Gesellschaft ärgerlichsten Aspekte betraf.

Im Juli 1937 wurde ein 'Komitee gegen Rassismus und Antisemitismus' gegründet. An seiner Gründung war ein sehr breites politisches Spektrum beteiligt (lediglich die Nationalisten fehlten): Mitglieder der Kommunistischen Partei unter Emilio Troise, führende Persönlichkeiten der Radikalen Partei wie Ricardo Balbín, auch Arturo Frondizi und Arturo Illia, die später Präsidenten werden sollten, sowie Vertreter konservativer Gruppen. Auch Persönlichkeiten aus dem kulturellen Bereich unterstützten das Komitee und verbreiteten die von ihm verfolgten Ziele, darunter Jorge Luis Borges, der vielleicht Bekannteste unter ihnen. ${ }^{3}$ Was diese so heterogene Gruppe verband, war die einhellige Verurteilung des italienischen Faschismus und des deutschen Nationalsozialismus. Sie interpretierten den Antisemitismus als ein Instrument, das von diesen Regimes benutzt wurde, um sich politischen Raum in der argentinischen Gesellschaft zu verschaffen. Der Kampf gegen Rassismus und Antisemitismus bedeutete für sie den Kampf für Demokratie und liberale Tradition in Argentinien. ${ }^{4}$

${ }^{2}$ V. Thermann 8.6.1940, A. A., Pol. IX, 619, Akten zur Deutschen Auswärtigen Politik, $1918-1945,437$.

${ }^{3}$ Actas del Primer Congreso Contra el Racismo y el Antisemitismo, Buenos Aires 6./7.8.1938, 10-13.

${ }^{4}$ Die Liga Argentina por los Derechos del Hombre schickte ein Telegramm an Dr. Le Breton - welcher zu diesem Zeitpunkt gerade als Vertreter Argentiniens an der Konferenz von Evian teilnahm - in dem unter anderem "eine positive Aktion zum Schutz der Menschen, die durch Intoleranz und Barbarei verfolgt werden" gefordert wurde, sowie ein weiteres Telegramm an den Vorsitzenden jener Konferenz: "die Menschen in der Welt müssen eine sichere Zuflucht im Schoße zivilisierter Nationen finden, egal welch politischer 
Am 6. und 7. August 1938 fand in den Hallen des Consejo Deliberante (Beratende Versammlung) der Stadt Buenos Aires der Erste Kongreß gegen Rassismus und Antisemitismus statt. In der vorbereitenden Kommission zum Kongreß arbeitete Dr. Alfredo Dang mit, ein sozialdemokratischer Abgeordneter, der aus Deutschland geflohen war. In Argentinien lehrte er als Dozent am Colegio Pestalozzi und war Gründungsmitglied der Vereinigung "Das Andere Deutschland". Arturo Frondizi, der auch die "Argentinische Liga für Menschenrechte' vertrat, verurteilte in der Eröffnungsrede die eingetretenen Veränderungen in der Einwanderungspolitik und begründete seine Ablehnung mit der Einschätzung, sie seien vollkommen verfassungswidrig und entfernten sich darüber hinaus von den in Argentinien verwurzelten Traditionen. In den Abschlußerklärungen forderte der Kongreß: "In diesen Augenblicken ist es dringend erforderlich, die Grenzen des Landes für die Flüchtlinge und Verfolgten aus Österreich, Deutschland, Rumänien und Polen zu öffnen, die zu Opfern einer erbarmungslosen rassischen Verfolgung geworden sind".

Bei verschiedenen Gelegenheiten griff eine der angesehensten Zeitungen Argentiniens die von der Regierung betriebene Einwanderungspolitik hart an. Diese Artikel in La Prensa fallen auf, weil sie sehr oft den Faktor Arbeitskraft ins Feld führen, um zu rechtfertigen, daß eine Ermöglichung der Immigration notwendig sei. So vertrat La Prensa die Ansicht, daß die Schließung der Grenzen den Verlust einer hervorragenden Gelegenheit bedeutete, hochqualifizierte Arbeitskräfte zu erhalten, die für das Land einen wichtigen Beitrag leisten könnten.

Im Nationalkongreß sprachen sich besonders die radikalen und sozialistischen Abgeordneten gegen das Dekret aus dem Jahre 1938 aus. Anders war dagegen die Haltung der Konservativen. Als im Abgeordnetenhaus über die Einrichtung einer Untersuchungskommission zur Aufklärung nationalsozialistischer Aktivitäten in Argentinien beraten wurde, schlug etwa der Abgeordnete Güiraldes die Schaffung einer anderen Art von Kommission vor: Sie sollte stattdessen die Aktivitäten von Gruppen untersuchen, die auf der Flucht aus Europa ins Land gekommen waren. In diesem Zusammenhang spricht er von einer "Existenz regelrechter Organisationen, die jüdische Immigranten in unser Land holen sollen, und immer wieder hören wir von den Schilderungen argentinischer Reisender, die stets übereinstimmend von einer ungeahnten Menge von Juden sprechen, die unter den Ankommenden sind [...], wogegen wir uns verteidigen müssen. Diese Neuankömmlinge, die sich zusammenschließen, die an ihrer überlieferten Religion und alten Gebräuchen festhalten, die ihre eigenen Schulen und Tempel bauen, die sich innerhalb einer Rasse abkapseln und

Einstellung, religiösen Glaubens oder sozialen Hintergrunds". Interview mit Dr. Arturo Frondizi, 2.9.1991. 
Andersrassige und -gläubige als fremd ansehen, diese Neuankömmlinge drohen uns schwerwiegende soziale und wirtschaftliche Probleme zu bringen". 5

Sozialistische Abgeordnete waren es, die zu diesem Zeitpunkt beantragten, die Haltung der deutschen Regierung gegenüber den Juden in den Debatten zu behandeln und die Grenzen Argentiniens zumindest für jüdische Kinder zu öffnen. Es war ebenfalls ein sozialistischer Deputierter, Enrique Dickmann, der es einer Gruppe von immigrierten deutschen Nazigegnern ermöglichte, ihre Ideen in einem Papier darzulegen, das im Kongreß verlesen wurde: Die genannte Gruppe hatte ein Hilfskomitee für jene ins Leben gerufen, die auf der Flucht aus Deutschland nach Argentinien kamen. Das Komitee erhielt den Namen "Das Andere Deutschland". Es ging der Gruppe darum zu zeigen, daß es Deutsche innerhalb und außerhalb Deutschlands gab, die den Nationalsozialismus ablehnten und die Methoden des Dritten Reiches bekämpften (s. dazu Seelisch 1969 und Mühlen 1988).

Sowohl auf parlamentarischer Ebene als auch in den Massenmedien war der Widerstand gegen die restriktiven Einwanderungsgesetze eng verbunden mit dem Kampf gegen die nationalsozialistischen Aktivitäten in Argentinien. Wie man später in den Debatten des Abgeordnetenhauses eingestand, war der Kampf gegen die nazistischen Aktivitäten in Argentinien von der Presse begonnen worden. Die argentinische Öffentlichkeit hatte die Gelegenheit, sich mittels der auflagenstärksten Zeitungen La Nación, La Prensa und Crítica von der Ausbreitung des Nationalsozialismus im Lande ein Bild zu machen, noch bevor sich die Regierung oder das Abgeordnetenhaus mit dem Thema befaßten. Bevor 1936 die Deutsche Diplomatische Vertretung den Status einer Botschaft erhielt, hatte sich der Delegationsführer bei der argentinischen Regierung wegen der von der Tageszeitung Crítica und dem Argentinischen Tageblatt geführten Kampagne gegen "das Deutsche" - wie er es nannte - beschwert, während es sich tatsächlich um eine Kampagne gegen die nationalsozialistische Propaganda gehandelt hatte (Bussemeyer 1938, 134).

Seit der Ausrufung Hitlers zum Kanzler im Januar 1933 hatte das Argentinische Tageblatt, die von Dr. Ernesto Alemann geleitete Zeitung der deutschen Gemeinde, in seinen Leitartikeln den Nationalsozialismus angegriffen. Diese Zeitung, die darüber hinaus vielen Immigranten Arbeit gab, prangerte beharrlich die in Deutschland verübten Verbrechen an und hörte nicht auf, die nazistischen Umtriebe in Argentinien publik zu machen und zu kritisieren; diese Linie behielt die Zeitung bis zum Zusammenbruch des Naziregimes in Deutschland

\footnotetext{
${ }^{5}$ Diario de Sesiones, Cámara de Diputados, 16.6.1939: 688. — In den nationalistischen Propagandareden gesellte sich zum Antisemitismus der Konflikt zwischen dem "jus solis" und dem "jus sanguinis", der den Abgeordneten Sánchez Viamonte mit einer deutlichen Anklage an die Adresse der deutschen Gemeinde in Argentinien sagen läßt: "In Argentinien gibt es ausländische Frauen, die keine argentinischen Kinder zur Welt bringen". Diario de Sesiones, Cámara de Diputados, 30.5.1940: 263.
} 
bei (vgl. Spitta 1990). Ihr Einflußbereich war aber auf die deutschsprachigen Leser beschränkt, so daß man nicht davon ausgehen kann, daß die von ihr vertretenen Ansichten die Gesellschaft insgesamt beeinflußten. Die deutsche Botschaft sagte später in einem Bericht: "Es sind nicht nur linke Zeitungen, die das Dritte Reich kritisieren. Im Namen von Demokratie und Freiheit haben sich seriöse Zeitungen wie La Nación, La Prensa oder die Abendzeitung La Razón massiv und auf das energischste gegen die Organisation von Deutschen und deutschen Schulen im Ausland ausgesprochen". ${ }^{6}$

1938 sprachen sich im Kongreß sozialistische und verschiedene radikale Abgeordnete klar gegen die Politik der Abschottung gegenüber Einwanderern aus. Mitte September des Jahres, noch bevor die neuen Verfügungen in Kraft traten, bat der Abgeordnete Juan Solari die Regierung um eine Erklärung zum Ausstellungsstop für Einreisegenehmigungen. Die Regierung führte in ihrer Antwort ähnliche, nur noch vager formulierte Argumente wie in der Dekretsbegründung an.

Man muß in diesem Zusammenhang hervorheben, daß die argentinischen Behörden gegenüber dem Flüchtlingsproblem eine zweideutige Politik vertraten. Sowohl bei internationalen Treffen wie auch bei der Rechtfertigung der diversen Vorschriften, die den Zustrom von Immigranten beschränkten - bevor die Grenzen des Landes definitiv geschlossen wurden - wurde die Absicht, die Einreise von Flüchtlingen aus dem Dritten Reich zu begrenzen, nie offen ausgesprochen. Sätze wie "Argentinien sieht voller Sympathie jegliche Initiative zur Aufnahme von Flüchtlingen im Land, sofern die Immigration nach den geltenden Gesetzen erfolgt" oder "wir sind der Überzeugung, daß wir mit unserer Haltung bei der Einwanderungsfrage in der Vergangenheit ausreichend die Großzügigkeit des Standpunktes bewiesen haben, den wir in Zukunft weiterhin einnehmen werden" oder "der Anteil von Juden in der argentinischen Bevölkerung zählt zu den höchsten in der Welt, und angesichts der binnenwirtschaftlichen Lage und der Situation auf dem Arbeitsmarkt können nur Landwirte aufgenommen werden"7 dienen der abgeschwächten Darstellung einer viel drastischeren Wirklichkeit, die in immer restriktiveren Dekreten zur Einreise von Flüchtlingen nach Argentinien deutlich wird.

Berücksichtigte die Regierung die öffentliche Meinung, welche sich gegen die Ausbreitung nationalistischer Aktivitäten aussprach und für eine flexiblere Einwanderungspolitik eintrat? Was die Aktivitäten von Nazigruppierungen

${ }^{6}$ Meynen, Buenos Aires 5.4.1938 an das Auswärtige Amt.

${ }^{7}$ Foreign Relations, 1928, vol. 1, 791, 799, 841, 842. Wie die Verantwortlichen der argentinischen Außenpolitik selbst zugaben, waren diese Argumente "Formeln, die es uns erlauben sollten, den Anschein der Kooperation bei den Aufgaben der Kommission (es handelt sich um das Internationale Flüchtlingskomitee, Anm. d. A.) zu wahren, ohne deshalb unsere Position im Kern zu gefährden". Brief von Felipe Aja Espil, argentinischer Botschafter in den USA, an den argentinischen Außenminister Dr. José M. Cantiló am 25.8.1939. - Archivo General del Ministerio de Relaciones Exteriores y Culto. 
anbelangte, traf man Maßnahmen zu deren Eindämmung. Aber gerechtfertigt wurden sie nicht mit den in Parlament und Massenmedien laut gewordenen Argumenten. Man kritisierte nicht den totalitären Charakter des nationalsozialistischen Gedankens, der mit den fundamentalen Grundsätzen der argentinischen Verfassung unvereinbar war, sondern es wurde die Notwendigkeit unterstrichen, daß die Führer sowie Abzeichen und Symbole der Vereinigungen argentinischer Herkunft sein müßten: ein nationalistisches Argument also. Außerdem machte man eine demokratische innere Organisation der Gruppen zur Bedingung.

Die öffentliche Meinung, die sich über die Mitte- und Linksparteien im Parlament und die wichtigsten Zeitungen im Land artikulieren konnte, stellte sich der immer offensichtlicheren Antiflüchtlingspolitik mit ihren Einwanderungsbeschränkungen entgegen. Sie wurde allerdings nicht berücksichtigt, und das Handeln der Regierung entsprach in dieser Hinsicht vielmehr nationalkonservativem Denken. Beiden so unterschiedlichen Regierungsmaßnahmen (Verbot der nationalsozialistischen Aktivitäten im Land und Verhängung eines Einreisestops für Flüchtlinge) lag dennoch ein vages Konzept von "nationaler Souveränität" zugrunde, das der nationalistischen Stimmung jener Zeit entsprach.

"Man muß der semitischen Invasion entgegentreten", schrieb die ultranationalistische Zeitung La Fronda; "die Aktivitäten der Nazis beinhalten antiargentinische Propaganda und belasten das Land", erklärte ein führender Politiker. Diese eindeutig nationalistischen Argumente wurden von der argentinischen Regierung berücksichtigt.

Zusammenfassend läßt sich sagen: Obwohl sich ein bedeutender Teil des politischen Spektrums und praktisch die gesamte Presse mit den Flüchtlingen des Dritten Reiches solidarisch erklärten, schlossen die argentinischen Behörden die Grenzen für Einwanderer. Die wirkliche Erklärung für diese politische Entscheidung liegt nicht in den so häufig angeführten wirtschaftlichen Problemen, sondern in dem damals herrschenden nationalistischen Klima, das paradoxerweise auch die von der Regierung unternommenen Anstrengungen zur Eindämmung der nationalsozialistischen Aktivitäten im Land erklärt.

Aus dem Spanischen von Maria Maier 


\section{Bibliographie:}

Avni, Haim. 1983. Argentina y la historia de la inmigración judia 1810-1950. Buenos Aires: Universidad Hebrea de Jerusalén.

Bussemeyer, Peter. 1938. 50 Jahre Argentinisches Tageblatt. Werden und Aufstieg einer Auslandsdeutschen Zeitung. Buenos Aires.

Jackisch, Carlota. 1987. El Nazismo y los Refugiados Alemanes en la Argentina. Buenos Aires: Editorial de Belgrano.

-. 1989. Die Einwanderungspolitik Argentiniens gegenüber den Juden 19331945. In: Schrader, Achim; Karl Heinrich Rengstorf (Hg.): Europäische Juden in Lateinamerika. St. Ingbert: Röhrig.

Jacobsen, Hans-Adolf. 1968. Nationalsozialistische Außenpolitik, 1933-1938. Frankfurt a.M., Berlin: Metzner.

Mühlen, Patrik von zur. 1988. Fluchtziel Lateinamerika - Die deutsche Emigration 1933-1945: Politische Aktivitäten und soziokulturelle Integration. Bonn: Neue Gesellschaft.

Seelisch, Winfried. 1969. Das Andere Deutschland. Eine politische Vereinigung deutscher Emigranten in Südamerika. Unveröffentlicht. Berlin: Otto SuhrInstitut.

Spitta, Arnold. 1990. Beobachtung aus der Distanz. Das Argentinische Tageblatt und der deutsche Faschismus. In: Koebner, Thomas (Hg.). Politische Aspekte des Exils. München: Ed. Text und Kritik. 